\title{
Determinantes e indicadores de la salud
}

\section{Determinants and health indicators}

\section{Ernesto Cotonieto-Martínez ${ }^{a}$}

\begin{abstract}
:
The determinants of health are a set of factors involved in the Illness-health process that must be included in the formulation and implementation of public policies. Meanwhile, the health indicators have the function of identifying the impact that these actions have had on the well-being among the population. Both are very important for decision-making because they provide information that allows to identify what actions must be carried out.
\end{abstract}

\section{Keywords:}

Determinants of Health, Health Indicators, Public Policies, Public Health, Evidence-Based Practice.

\section{Resumen:}

Los determinantes de la salud son un conjunto de factores involucrados en el proceso salud-enfermedad que deben ser incluidos en la formulación e implementación de políticas públicas. Mientras que los indicadores de salud tienen la función de identificar el impacto que esas acciones han tenido sobre el bienestar de la población. Ambos son muy importantes para la toma de decisiones porque proporcionan información que permite reconocer qué acciones deben llevarse a cabo.

\section{Palabras Clave:}

Determinantes de la Salud, Indicadores de Salud, Políticas Públicas, Salud Pública, Práctica Basada en Evidencia.

\section{Introducción}

Los determinantes de la salud son un conjunto de condiciones en las que una persona nace, crece, trabaja, vive, envejece y que inciden en el proceso saludenfermedad a individual y colectivo se encuentra determinado por una gran cantidad de factores a un nivel micro, meso, macro sistema ${ }^{1-2}$.

Estas condiciones, rebasan al modelo biomédico y reduccionista sobre la salud, por lo que incluyen a las estructuras y sistemas en materia política, social, ambiental, laboral, seguridad, alimentaria, religiosa, educativa, económica, cultural, legislativa, entre otras ${ }^{1-2} y$ que afectan tanto el funcionamiento como la calidad de vida de las personas ${ }^{1-3}$.
En tanto que, de acuerdo con la World Health Organization, los indicadores de salud están conformados por un conjunto de indicadores básicos que sirven para determinar y atender las prioridades en salud y que incluyen 100 indicadores distribuidos en cuatro dominios ${ }^{3}$ :

Estado de salud, Factores de riesgo. Cobertura de servicio y Sistemas de salud. Información en salud, seguridad en salud, equidad.

\section{¿Qué relación tienen?}

El estudio de los determinantes de la salud es de gran importancia para comprender, identificar e intervenir en los factores que están afectando la salud de una población y fortalecer aquellos que representan una protección ante el origen y mantenimiento de las enfermedades.

\footnotetext{
a Autor de Correspondencia. Universidad Autónoma del Estado de Hidalgo. Instituto de Ciencias de la Salud. ORCID:
} 

Hidalgo, Vol. 9, No. 18 (2021) 74-76

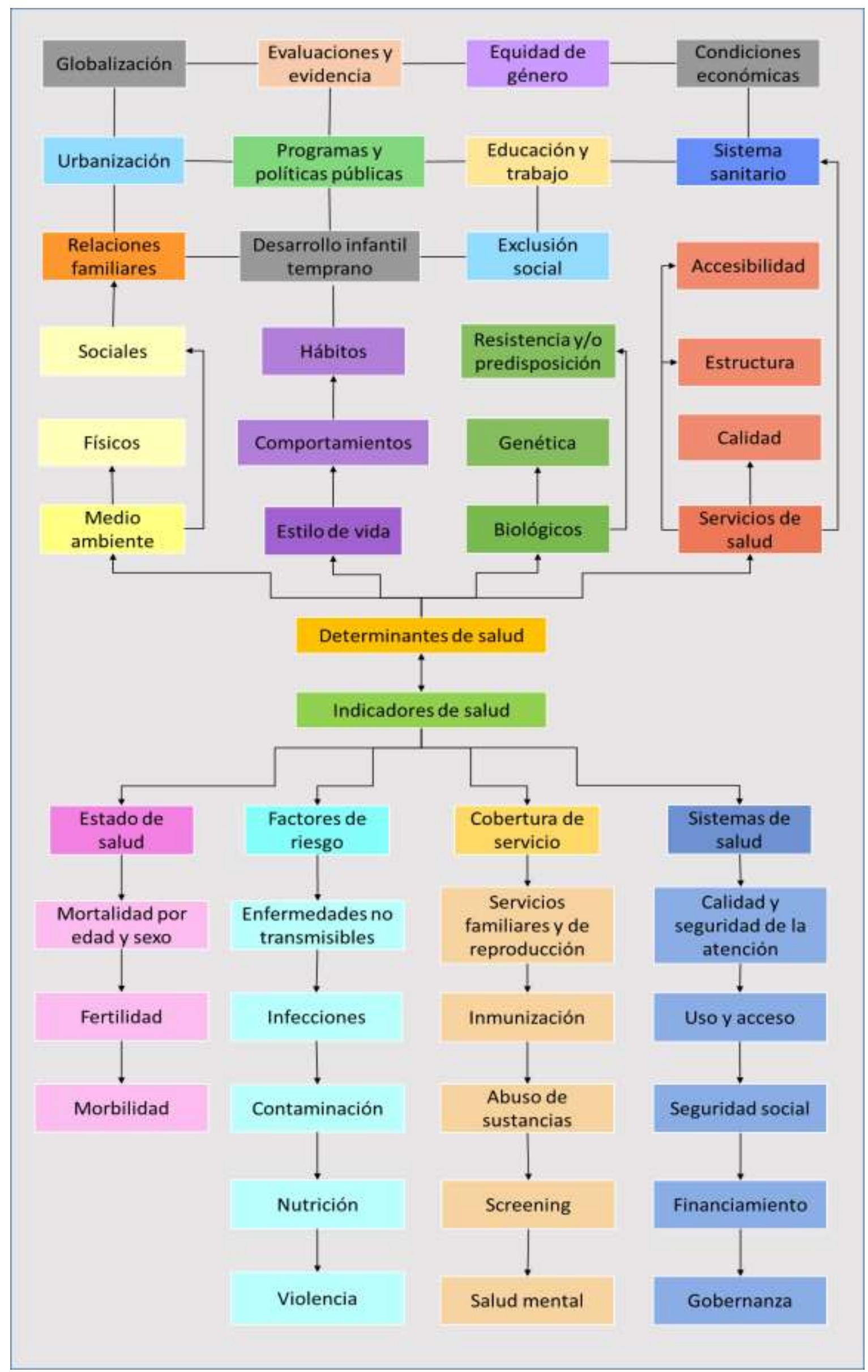


Esa es la premisa que debe seguirse en materia de diseño de políticas públicas (lo que incluye al Gobierno, la sociedad civil y otros agentes involucrados); deben formularse acciones basadas en información sobre las condiciones en que se da el proceso salud-enfermedad en un contexto determinado.

Esto es muy importante ya que, de acuerdo con la experiencia internacional, las políticas y programas públicos implementados bajo enfoques integrales y ecológicos tienen una mayor probabilidad de incidir en la salud de la población.

En este sentido, los indicadores de salud permiten recolectar información sobre qué tan eficaces han sido las acciones que se han llevado a cabo, saber en qué medida la realidad del proceso salud-enfermedad ha sido afectada y si este impacto ha sido positivo o negativo. De tal forma que permitan tomar decisiones respecto a su suspensión, mantenimiento o apuntalamiento.

Es decir, los indicadores deben ser vistos como el fiel reflejo de qué tanto las políticas están modificando la carga de los determinantes de la salud, a la vez que estos deben ser considerados en la construcción de dichas políticas y de sus respectivos indicadores.

De otro modo, las acciones difícilmente tendrán la eficacia deseada y los resultados de su medición no serán congruentes con la realidad; lo que implicaría un gasto infértil de recursos, como generalmente sucede en países que no basan sus decisiones en evidencia científica ni gestionan lo necesario para medir de manera adecuada sus intervenciones.

\section{Referencias}

[1] World Health Organization. Social determinants of health. [Internet]. s.f. [Consultado 25 mayo 2020]. Disponible en: https://www.who.int/social_determinants/en/

[2] Office of Disease Prevention and Health Promotion. Understanding Social Determinants of Health. [Internet]. 2020. [Consultado 15 mayo 2020]. Disponible en: https://www.healthypeople.gov/2020/topicsobjectives/topic/social-determinants-of-health

[3] World Health Organization (2018). Global Reference List of 100 Core Health Indicators (plus health-relates SDGs. [Internet] 2018. [Consultado 27 mayo 2020]. Disponible en: https://www.who.int/healthinfo/indicators/2015/metadata/en/ 